\title{
EP-131
}

\section{Bile leakage from subvesical bile duct (duct of Luschka) after laparoscopic cholecystectomy}

\author{
Young-Min KIM, Gun-Hyung NA, II-Young PARK*
}

Department of Surgery, Catholic University of Korea, Bucheon St. Mary Hospital, Bucheon, Korea

Introduction: Bile leakage is a serious complication in laparoscopic cholecystectomy. Subvesical bile duct (duct of Luschka) injury is a secondary major cause of bile duct injury after laparoscopic cholecystectomy.

Methods: Between January 2019 and December 2020, we performed 1,269 laparoscopic cholecystectomies in our department. There were 4 cases $(0.3 \%)$ of subvesical bile duct injury. 1 case was found during operation, the other 3 cases developed abdominal pain after postoperative 3 th or 7 th days.

Results: In the intra-operative case, the injured duct was closed with endo-clip. There was no post-operative complication. Postoperatively found cases were treated with endoscopic retrograde cholagiopanreatography (ERCP). However, ERCP failed (2), continuous bile leak and abdominal pain (1) developed. Re-laparoscopy (2) and explo-laparotomy (1) performed.

Conclusions: During operation, meticulous observation of the gallbladder fossa should be performed after cholecystectomy. If bile leak has found post-operatively, ERCP with biliary stenting is effective. If ERCP fails, surgical treatments required. A surgeon should be aware of this anomaly of the bile duct and know about the prevention and the managements. 